\title{
As contribuições do jogo de RPG no ensino médio: o que dizem as produções científicas brasileiras
}

Luciano da Silva Rodrigues, IFRS/MPIE Campus Porto Alegre, lucianodsradm@gmail.com - Orcid: https://orcid.org/0000-0001-8332-6783

Marcelo Augusto Rauh Schmitt, IFRS/MPIE Campus Porto Alegre, marcelo.schmitt@poa.ifrs.edu.br - Orcid: https://orcid.org/0000-0003-1290-5029

Silvia de Castro Bertagnolli, IFRS/MPIE Campus Porto Alegre, silvia.bertagnolli@ poa.ifrs.edu.br - Orcid: https://orcid.org/0000-0001-7495-6636

Resumo: Um dos problemas enfrentados atualmente na escola básica é a desmotivação e a falta de engajamento dos estudantes. Uma das estratégias pedagógicas apontadas como solução compreende o uso de jogos, sendo que o gênero de Role Playing Game (RPG) pode ser usado para desenvolver diferentes habilidades. Assim, foi realizada uma investigação, no formato de um mapeamento sistemático, com o objetivo de prover uma visão geral das pesquisas a respeito de jogos do gênero de RPG e identificar as contribuições para o ensino médio no Brasil. Como resultados principais, identificou-se que os jogos RPGs podem ser usados como uma alternativa pedagógica em sala de aula, possibilitando abordar diversos cenários e narrativas, integrando diferentes áreas do conhecimento e explorando diversas habilidades dos estudantes.

Palavras-chave: Aprendizagem baseada em jogos. Role Playing Game (RPG). Ensino Médio.

\section{RPG contributions in high school: what Brazilian scientific productions affirm}

Abstract. Currently, one faced problem in Brazilian K12 schools is a lack of student motivation and engagement. Using games as a pedagogical strategy is considered as one possible solution to this problem and Role-Playing Games are used to develop different skills. Thus, a systematic mapping investigation was carried out. This review aims to provide an overview of research on RPG and their contributions to Brazilian high school. As main results, we identified that RPGs can be used as a pedagogical alternative in the classroom. RPGs allow to approach different scenarios and narratives and can be applied in different areas of knowledge, and explore different skills of students.

Keywords: Game-based learning. Role-Playing Game (RPG). K-12 education.

\section{Introdução}

Nos últimos anos, algumas pesquisas têm apontado que existem vários fatores que contribuem para a evasão no ensino médio (Gallup, 2016; Insper, 2017). Segundo dados apontados em Insper (2017), há três causas principais: contexto (fatores externos ao ambiente escolar que influenciam o abandono); compreensão da real importância da escola e da educação, e seus impactos na vida; e motivação (alguns jovens não percebem o significado dos conteúdos abordados e a sua relação com os problemas do cotidiano).

No que diz respeito ao terceiro fator citado, é importante ressaltar que, muitas vezes, docentes encontram dificuldades para engajar seus alunos durante a aula, e até mesmo na realização de atividades em grupo. Tal fato deve ser alvo de grande atenção já que, de acordo com Keller (2009), o discente deve estar motivado para aprender, para que a aprendizagem de fato se efetive. Como alternativa para superar esta questão, recursos tecnológicos tais como redes sociais, objetos de aprendizagem, jogos digitais e aplicativos 
podem ser utilizados pelo docente, de forma coerente com o seu planejamento pedagógico, para incentivar o interesse e o engajamento do aluno.

Visando a aumentar o engajamento dos estudantes, algumas pesquisas relatam que a utilização de jogos nos processos de ensino e de aprendizagem pode ser uma alternativa, devido ao seu potencial de mobilizar os estudantes para realizarem atividades propostas no contexto escolar (Savi; Ulbricht, 2008; Mayo, 2009; Santos; Isotani, 2018; Meira; Blinkstein, 2020). Autores como Prensky (2001) e McGonigal (2011) descrevem que jogos, além de auxiliarem no envolvimento do discente, podem ser utilizados como recurso pedagógico para trabalhar qualquer área de conhecimento contida no currículo escolar, além de competências tais como tomada de decisão, comunicação, sociabilização, entre outras necessárias para conviver na sociedade. $\mathrm{O}$ uso de jogos educacionais, sejam eles digitais ou analógicos, corresponde a uma das estratégias pedagógicas utilizadas para favorecer o engajamento dos discentes e podem auxiliar no trabalho do professor, despertando o interesse dos estudantes (Sobreira; Viveiro; Abreu, 2020).

Os jogos possuem diversas classificações: esportes, luta, aventura, tiro, estratégia, simuladores, Role Playing Game (RPG), passatempo, entre outros. Um dos gêneros que têm sido adotado em estratégias pedagógicas é o denominado Role Playing Game ou RPG. Esse tipo de jogo utiliza dois elementos principais: a narrativa e a representação de papéis (Bittencourt; Giraffa, 2003, p. 684). O RPG utiliza-se da ludicidade para proporcionar um ambiente que estimula o estudante a pensar sobre suas ações (SOUZA, 2015). É preciso planejá-las uma vez que isso pode impactar as próximas "jogadas".

Ao incentivar a tomada de decisão colaborativa, a representação de um papel permite simular uma situação que seria difícil ou até arriscada na vida real, assim como colocar-se no lugar do outro. O uso de RPG pode contribuir para o desenvolvimento das habilidades descritas na Base Nacional Comum Curricular (BNCC), tais como raciocínio lógico, comunicação, cultura digital e repertório cultural.

Assim, considerando todos esses elementos, foi elaborado um mapeamento sistemático das publicações usando os passos estabelecidos por Kitchenham e Charters (2007), tendo como período de análise os anos de 2016 a 2020, com o objetivo de proporcionar uma visão geral das investigações científicas referentes à utilização de jogos do tipo RPG e suas contribuições para o ensino médio brasileiro. A delimitação realizada, com apenas publicações brasileiras, deve-se ao fato de que se deseja investigar as práticas que estão sendo realizadas conforme a realidade educacional do próprio país. Nessa busca, para cada pesquisa identificada, foram analisados alguns itens: competências dos estudantes, possibilidades de uso do RPG e resultados relatados com a sua aplicação, além das teorias de aprendizagem e das TICs (Tecnologias da Informação e Comunicação) utilizadas em sua aplicação.

Este artigo encontra-se organizado da seguinte forma: a seção 2 expõe o percurso metodológico para a sua realização e a sua sistematização; na seção 3, são apresentados as discussões e os resultados; e, por fim, são expostas as conclusões relacionadas ao presente trabalho.

\section{Percurso Metodológico}

Foi utilizado como percurso metodológico um mapeamento sistemático, que tem o objetivo de mapear estudos que possuem relação com um determinado tópico que se deseja investigar. Para realizá-lo foram seguidas as seguintes etapas (Kitchenham, 2004): (i) o planejamento, momento em que foram definidas as questões de pesquisa, a string de busca, a definição de critérios de inclusão e exclusão e quais bases seriam utilizadas para realizar a investigação; (ii) a condução da pesquisa a partir da chave de busca, a qual foi aplicada em algumas bases. Uma lista de estudos foi relacionada, utilizando-se um conjunto de RENOTE 
estudos primários. Para (Kitchenham; Charters, 2007), o estudo primário entrega uma evidência direta sobre as questões de pesquisa definidas, em seguida critérios de inclusão e exclusão foram utilizados; e, (iii) a análise sistematizada dos resultados, que visa a desenvolver esquemas de classificação em categorias e analisar os respectivos resultados com o objetivo de responder ao questionamento de pesquisa e corroborar com o pesquisador a tomar uma decisão em relação ao tema escolhido.

\subsection{Planejamento da Pesquisa}

A etapa do planejamento é muito importante, pois é nela que ocorrem as definições do objetivo da pesquisa e do conjunto de questões que permitem atingi-lo (Figura 1). Destaca-se que, para atingir o objetivo delimitado, foram analisados trabalhos que abordam RPG digital e híbrido, ou seja, pesquisas que desenvolveram o RPG usando algum tipo de tecnologia em alguma parte dele. Além disso, outro elemento essencial nessa etapa compreende a definição da string de busca, a qual foi estruturada partindo do objetivo da pesquisa e das questões elaboradas. É importante ressaltar que o termo Role Playing Game obteve o mesmo resultado de buscas que sua sigla RPG.

Figura 1 - Objetivo e Questões de Pesquisa, String de Busca

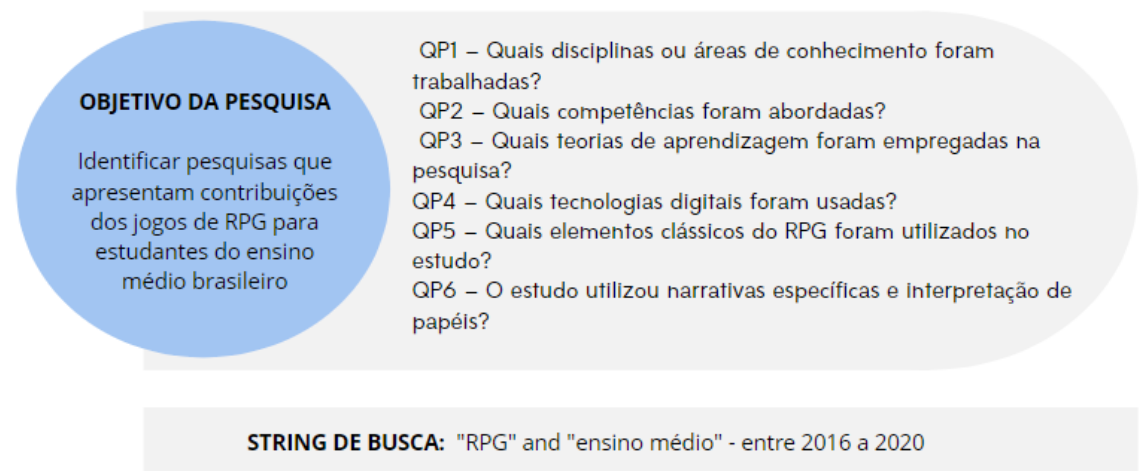

Fonte: Elaborado pelos autores, 2021.

Os critérios de inclusão e exclusão desta investigação foram definidos para auxiliar a responder às Questões de Pesquisa $(\mathrm{QP})$ ilustradas na Figura 1. Os critérios de inclusão permitiram que apenas estudos relacionados com o objetivo proposto por esta pesquisa fossem inseridos na análise dos resultados, são eles: (i) o estudo aplicou um jogo de RPG no contexto do ensino médio brasileiro; (ii) apresenta resultados dessa aplicação; e (iii) é um artigo completo publicado em evento ou periódico, podendo ser uma Tese de Doutorado ou Dissertação de Mestrado. Os critérios de exclusão contribuíram para remover artigos que não estão relacionados com o intento da investigação: (i) o estudo é um Resumo, PPP de curso, projeto, relatório, trabalho de conclusão de curso, monografia de especialização, ou capítulo de livro; (ii) a pesquisa não foi aplicada com estudantes do ensino médio brasileiro ou não foi testado; (iii) a investigação aborda RPG não digital, ou seja, sem o uso de nenhum tipo de TDIC; e (iv) o estudo não está disponível para download ou tem acesso restrito, se caracteriza como secundário ou foi localizado em outra base de dados.

Convém observar que foram investigadas as seguintes bases nacionais: Catálogo de Teses e Dissertações da CAPES, a Revista Novas Tecnologias na Educação (RENOTE), Google Acadêmico e o Portal do SBGames (https://www.sbgames.org/). Optou-se por essas bases pois são as que concentram as publicações da área de informática na educação. Embora a última escolha possa parecer mais restrita do que as outras, há que se ressaltar 
que se trata de evento brasileiro que concentra publicações relacionadas ao tema de jogos, no período de 2016 a 2020.

\subsection{Condução da Pesquisa}

Na etapa da condução da pesquisa, houve a busca de trabalhos nas bases de dados selecionadas. Na primeira busca realizada com a utilização da string de busca definida na Figura 1, considerando o período de 2016 a 2020, foram identificados 1720. A partir daí, procedeu-se à leitura dos seus títulos e resumos, assim como à verificação dos critérios de exclusão listados. Ao concluir essa análise, permaneceram 128 estudos cujas introdução, metodologia e conclusão foram lidas. Desta seleção, foram elencados 23 trabalhos (Quadro 1) que foram lidos na íntegra e analisados de forma detalhada em conformidade com objetivo planejado para essa pesquisa.

Quadro 1 - Artigos selecionados para análise

\begin{tabular}{|c|c|c|}
\hline ID $^{1}$ & Base & Título \\
\hline $\begin{array}{l}\text { E1 } \\
\text { D }\end{array}$ & $\begin{array}{l}\text { Google } \\
\text { Acadêmico }\end{array}$ & $\begin{array}{l}\text { MELO, C. V.; BERTAGNOLLI, S. C.; TONELLO, G. H. Do livro ao RPG: } \\
\text { conexões entre arte literária e jogos digitais em educação. \#Tear: Revista de } \\
\text { Educação, Ciência e Tecnologia, Canoas, v. 5, n. 2, dez., } 2016 .\end{array}$ \\
\hline $\begin{array}{l}\text { E2 } \\
D\end{array}$ & $\begin{array}{l}\text { CAPES Teses } \\
\text { e Dissertações }\end{array}$ & $\begin{array}{l}\text { SOUZA, A. L. P. RPG digital instrumento pedagógico para o ensino da } \\
\text { abolição da escravidão na Bahia. Salvador: Universidade do Estado da Bahia, } \\
\text { 2016. 122p. Dissertação de Mestrado. }\end{array}$ \\
\hline $\begin{array}{l}\text { E3 } \\
\text { D }\end{array}$ & $\begin{array}{l}\text { CAPES Teses } \\
\text { e Dissertações }\end{array}$ & $\begin{array}{l}\text { SILVA, C. A. C. O jogo de RPG digital como material potencialmente } \\
\text { significativo para a aprendizagem de conceitos em cinemática. Curitiba: } \\
\text { Universidade Federal do Paraná, 2017. 109p. Dissertação de Mestrado. }\end{array}$ \\
\hline $\begin{array}{l}\text { E4 } \\
\text { D }\end{array}$ & $\begin{array}{l}\text { CAPES Teses } \\
\text { e Dissertações }\end{array}$ & $\begin{array}{l}\text { SILVA, W. P. Jogos digitais adaptados para estudantes com deficiência visual: } \\
\text { estudo das habilidades cognitivas no Dosvox. Brasília: Universidade de } \\
\text { Brasília, 2017. 145p. Dissertação de Mestrado. }\end{array}$ \\
\hline $\begin{array}{l}\text { E5 } \\
\text { D }\end{array}$ & $\begin{array}{l}\text { CAPES Teses } \\
\text { e Dissertações }\end{array}$ & $\begin{array}{l}\text { OLIVEIRA, J. C. Jogo RPG (Role Playing Game) digital "Luta e Resistência": } \\
\text { uma proposta pedagógica para o ensino da história das rotas do tráfico de } \\
\text { escravizados na Bahia. Salvador: Universidade do Estado da Bahia, } 2017 . \\
\text { 190p. Dissertação de Mestrado. }\end{array}$ \\
\hline $\begin{array}{l}\text { E6 } \\
\text { D }\end{array}$ & $\begin{array}{l}\text { Revista } \\
\text { (RENOTE) }\end{array}$ & $\begin{array}{l}\text { FERREIRA JUNIOR, S. A. et al. Metodologias de uso de jogo computacional } \\
\text { RPG em sala de aula: estudo de caso com o jogo PeaceMaker. RENOTE, Porto } \\
\text { Alegre, v. } 15 \text {, n.1, jul., } 2017 \text {. }\end{array}$ \\
\hline $\begin{array}{l}\text { E7 } \\
\text { D }\end{array}$ & $\begin{array}{l}\text { CAPES Teses } \\
\text { e Dissertações }\end{array}$ & $\begin{array}{l}\text { AMORIM, D. C. Produção e uso de jogo digital RPG na formação inicial de } \\
\text { professores de biologia no contexto da aprendizagem baseada em jogos } \\
\text { digitais: possibilidades e desafios. Maceió: Universidade Federal de Alagoas, } \\
\text { 2017. 176p. Dissertação de Mestrado. }\end{array}$ \\
\hline $\begin{array}{l}\text { E8 } \\
D\end{array}$ & $\begin{array}{l}\text { Google } \\
\text { Acadêmico }\end{array}$ & $\begin{array}{l}\text { FELBER, D.; KRAUSE, J.; VENQUIARUTO, L. O uso de jogos digitais } \\
\text { como ferramenta de auxílio para o ensino de Física. Revista Insignare Scientia } \\
\text { - RIS, v. 1, n. 2, agosto, } 2018 \text {. }\end{array}$ \\
\hline $\begin{array}{l}\text { E9 } \\
\text { D }\end{array}$ & $\begin{array}{l}\text { Google } \\
\text { Acadêmico }\end{array}$ & $\begin{array}{l}\text { FERRARI, B. D. N.; MARTINS, R. L.; JUNIOR, N. K. JOGOS DIGITAIS } \\
\text { MULTIMODAIS E RPG: Experiências no desenvolvimento da consciência } \\
\text { ambiental a partir de recursos educacionais lúdicos. Revista Observatório, v. 4, } \\
\text { n. } 4 \text {, p. 201-228, junho, } 2018 \text {. }\end{array}$ \\
\hline $\begin{array}{l}\text { E10 } \\
\text { D }\end{array}$ & $\begin{array}{l}\text { CAPES Teses } \\
\text { e Dissertações }\end{array}$ & $\begin{array}{l}\text { CABALLERO, S. S. X. Modelo de análise socioconstrutivista para } \\
\text { compreensão do processo de aprendizagem mediado pelo Role Playing Game } \\
\text { (RPG) digital. Salvador: Universidade Federal da Bahia, 2018. 231p. Tese de } \\
\text { Doutorado. }\end{array}$ \\
\hline $\begin{array}{l}\text { E11 } \\
\text { D }\end{array}$ & $\begin{array}{l}\text { Google } \\
\text { Acadêmico }\end{array}$ & $\begin{array}{l}\text { SILVA, H. P. Desenvolvimento de um jogo de computador educacional de } \\
\text { Matemática - o mestre de Trigonometria. Seropédica: Universidade Federal } \\
\text { Rural do Rio de Janeiro, 2018.93p. Dissertação de Mestrado. }\end{array}$ \\
\hline
\end{tabular}

\footnotetext{
${ }^{1}$ Nesta coluna será indicada uma identificação única para cada estudo e uma legenda que determina se o jogo é Digital (D) ou Híbrido (H).

V. $19 \mathrm{~N}^{\circ} 2$, Dezembro, 2021

DOI: https://doi.org/10.22456/1679-1916.121188 


\begin{tabular}{|c|c|c|}
\hline $\begin{array}{l}\text { E12 } \\
\text { D }\end{array}$ & $\begin{array}{l}\text { Revista } \\
\text { (RENOTE) }\end{array}$ & $\begin{array}{l}\text { SILVA, D. P.; BEZ, M. R.; RIGO, S. J. My TechLife: Desenvolvimento e } \\
\text { Validação de um Jogo para o Ensino Técnico em Informática. Revista Novas } \\
\text { Tecnologias na Educação (RENOTE), Porto Alegre, v. 16, n.1, julho, } 2018\end{array}$ \\
\hline $\begin{array}{l}\text { E13 } \\
\text { D }\end{array}$ & $\begin{array}{l}\text { Google } \\
\text { Acadêmico }\end{array}$ & $\begin{array}{l}\text { CLASSE, T.M.; ALMEIDA, V. H.; FARIA, D. S. L. The ProUni Game - } \\
\text { ensinando o processo de solicitação do ProUni através de um jogo digital. In: } \\
\text { Seminário Jogos Eletrônicos Educação Comunicação, 8., 2019, Salvador. } \\
\text { Anais [...]. Salvador: UNEB. p. 160-170. }\end{array}$ \\
\hline $\begin{array}{l}\text { E14 } \\
\text { D }\end{array}$ & $\begin{array}{l}\text { Google } \\
\text { Acadêmico }\end{array}$ & $\begin{array}{l}\text { KAKPO, A. J. Anotação e transcrição funcional em sala de aula para a } \\
\text { aquisição de vocabulário. Revista Intercâmbio, São Paulo, v. 41, n.1, p. 1-24, } \\
\text { nov., 2019. }\end{array}$ \\
\hline $\begin{array}{l}\text { E15 } \\
\text { D }\end{array}$ & $\begin{array}{l}\text { CAPES Teses } \\
\text { e Dissertações }\end{array}$ & $\begin{array}{l}\text { MATOS, M. M. Ensino de Astronomia com aprendizagem baseada em game: } \\
\text { o caso Bendegó. Araguaína: Universidade Federal do Tocantins, 2020. 61p. } \\
\text { Dissertação de Mestrado. }\end{array}$ \\
\hline $\begin{array}{l}\text { E16 } \\
\mathrm{H}\end{array}$ & $\begin{array}{l}\text { Google } \\
\text { Acadêmico }\end{array}$ & $\begin{array}{l}\text { MACHADO, P. A. P.; SILVA, J. P. M.; SANTOS, L. M. A.; BARIN, C. S. } \\
\text { Utilizando RPG (Role-Playing Game) no Ensino de Matemática para alunos do } \\
\text { Ensino Médio. In: Anais do } 1^{\circ} \text { Compartilhando Saberes. Universidade Federal } \\
\text { de Santa Maria. v. 1, n. } 1,2017 \text {. }\end{array}$ \\
\hline $\begin{array}{l}\text { E17 } \\
\mathrm{H}\end{array}$ & $\begin{array}{l}\text { CAPES Teses } \\
\text { e Dissertações }\end{array}$ & $\begin{array}{l}\text { POOL, M. A. P. Desafios educacionais criativos associados às práticas } \\
\text { docentes: estudo de caso considerando RPG educacional. Porto Alegre: } \\
\text { Pontifícia Universidade Católica do Rio Grande do Sul, 2017. 178p. Tese de } \\
\text { Doutorado. }\end{array}$ \\
\hline $\begin{array}{l}\text { E18 } \\
\mathrm{H}\end{array}$ & $\begin{array}{l}\text { CAPES Teses } \\
\text { e Dissertações }\end{array}$ & $\begin{array}{l}\text { ALVES, V. O. Promoção de alfabetização científica em sentido estendido por } \\
\text { meio do desenvolvimento de um Role-Playing Game (RPG). Lorena: Escola } \\
\text { de Engenharia de Lorena, 2018. 141p. Dissertação de Mestrado. }\end{array}$ \\
\hline $\begin{array}{l}\text { E19 } \\
\mathrm{H}\end{array}$ & $\begin{array}{l}\text { CAPES Teses } \\
\text { e Dissertações }\end{array}$ & $\begin{array}{l}\text { OLIVEIRA, J. R. Games digitais: uma abordagem de física de partículas } \\
\text { elementares no ensino médio. Brasília: Universidade de Brasília, 2018. 158p. } \\
\text { Dissertação de Mestrado. }\end{array}$ \\
\hline $\begin{array}{l}\text { E20 } \\
\mathrm{H}\end{array}$ & $\begin{array}{l}\text { CAPES Teses } \\
\text { e Dissertações }\end{array}$ & $\begin{array}{l}\text { COSTA JÚNIOR, F. V. Uso do jogo "marinheiros do espaço” como } \\
\text { ferramenta pedagógica nas aulas de astronomia do ensino médio. Mossoró: } \\
\text { Universidade Federal Rural do Semi-Árido, 2019. 118p. Dissertação de } \\
\text { Mestrado. }\end{array}$ \\
\hline $\begin{array}{l}\text { E21 } \\
H\end{array}$ & $\begin{array}{l}\text { CAPES Teses } \\
\text { e Dissertações }\end{array}$ & $\begin{array}{l}\text { LIMA JUNIOR, G. R. Realidade aumentada: uma abordagem dinâmica no } \\
\text { ensino do meio ambiente para estudantes da educação básica. Maceió: Centro } \\
\text { Universitário Cesmac, 2019. 164p. Dissertação de Mestrado. }\end{array}$ \\
\hline $\begin{array}{l}\text { E22 } \\
H\end{array}$ & $\begin{array}{l}\text { Google } \\
\text { Acadêmico }\end{array}$ & $\begin{array}{l}\text { MENDES, L. F. O.; BOECHAT, L. T.; GARZONI, L. C. Trabalho nos } \\
\text { extremos: uma reflexão sobre trabalho e guerra fria utilizando técnicas de RPG } \\
\text { no ensino médio integrado. REPECULT- Revista Ensaios e Pesquisa em } \\
\text { Educação e Cultura, Seropédica, v. 5, n. 8, p. 154-189, jun, } 2020 \text {. }\end{array}$ \\
\hline $\begin{array}{l}\text { E23 } \\
H\end{array}$ & $\begin{array}{l}\text { Google } \\
\text { Acadêmico }\end{array}$ & $\begin{array}{l}\text { LIMA, F. M. Identificando práticas epistêmicas na utilização de um jogo sério } \\
\text { colaborativo para o ensino de imunologia. Belo Horizonte: Universidade } \\
\text { Federal de Minas Gerais, 2020.96p. Dissertação de Mestrado. }\end{array}$ \\
\hline
\end{tabular}
Fonte: Elaborado pelos autores, 2021.

Depois de aplicar os critérios de exclusão, foram selecionados 23 estudos nacionais para serem analisados na presente pesquisa.

\section{Discussões e resultados}

Ao término da leitura dos 23 estudos selecionados, estes foram analisados em detalhes com o propósito de elucidar as questões de pesquisa presentes na Figura 1 e o objetivo deste estudo. A primeira questão da pesquisa, "Quais disciplinas ou áreas de conhecimento foram trabalhadas?", tem como objetivo determinar quais são as disciplinas do ensino médio que mais utilizam jogos do gênero RPG em seus processos de aprendizagem, visando a identificar se a proposição desse tipo de estratégia privilegia abordagens interdisciplinares. Como exemplos de interdisciplinaridade pode-se citar os seguintes trabalhos: E17, que integrou uma variedade de áreas: matemática, física, química, história, geologia, botânica, geografia e navegação; E21, que abordou Educação 
ambiental e Geografia; E6 e E10, que relacionaram no RPG Geografia e História; E9, E18 e E21, que deram ênfase sobre a importância da preservação ambiental; E15 e E20, que aplicaram o conteúdo de Astronomia; E2 e E5, que abordaram a Escravidão ocorrida no Brasil; E7, que trataram de Biologia com concentração em Citologia. Com essa análise, pode-se perceber que a maior parte dos trabalhos foi desenvolvida em áreas que possuem narrativas de fatos, temas ou cenários históricos, como ciências, história, geografia, cidadania, democracia digital e educação. Observa-se que oito estudos (E3, E8, E11, E15, E16, E18-E20) abordaram temas vinculados às áreas de física, matemática e química.

Considerando a segunda questão da pesquisa, "Quais competências foram abordadas?", observou-se que o trabalho em equipe, a resolução de problemas, o apoio à tomada de decisões, a conscientização, a colaboração, o pensamento crítico e a criatividade foram as competências mais citadas nesses trabalhos. Um outro ponto que merece destaque são as argumentações apresentadas em E3 e E18, de que com o uso do RPG ficou mais claro para os estudantes a percepção quanto à aplicabilidade dos conteúdos em uma atividade simulada, construída a partir de fenômenos reais, e de que foi possível perceber a alfabetização científica dos participantes. Os estudos analisados ainda argumentam que o desenvolvimento de algumas habilidades e competências pode ser propiciado pelo uso do RPG, como, por exemplo, "formação de equipe por meio de aprendizagem em conjunto; e reflexão crítica e conexão do jogo com conhecimentos formais" (E2); podem ser usadas para introduzir "os estudantes em um ambiente colaborativo, participativo e de aprendizagem significativa" (E21). Em consonância com isso, pode-se usar uma das argumentações encontradas em E19 que descreve "que uma aprendizagem baseada em jogos digitais está de acordo com as necessidades e estilos de aprendizagem desta geração e das futuras, além de ser motivadora porque é divertida. Houve muita cooperação entre os alunos, mesmo sendo a premiação apenas para o melhor." Tomando-se como base todas as conclusões dos estudos selecionados, pode-se afirmar que essa abordagem estimula o desenvolvimento de habilidades e competências diversas, assim como incentiva a aprendizagem de uma forma motivadora, ancorando-se na ludicidade.

Quanto à terceira questão da pesquisa, "Quais teorias de aprendizagem foram empregadas na pesquisa?", constatou-se que as teorias de aprendizagem servem como base para o docente conhecer as diferentes concepções sobre aprendizagem e, então, transformar este conhecimento teórico de acordo com sua realidade para favorecer o desenvolvimento do conhecimento dos discentes. Na maioria dos trabalhos analisados, alguma teoria de aprendizagem foi especificada. Em alguns casos, o trabalho fundamentou-se em uma determinada teoria (E1-E5, E8-E10, E13, E15-E20 e E22), em outros, a teoria da aprendizagem foi apenas citada sem mencionar como foi aplicada ao estudo em si. O Gráfico 1 ilustra o percentual de distribuição dos teóricos da aprendizagem nos estudos analisados. Nota-se que os aspectos teóricos vinculados às teorias de Vygotsky e Paulo Freire foram os que mais subsidiaram as decisões metodológicas do uso do RPG. 


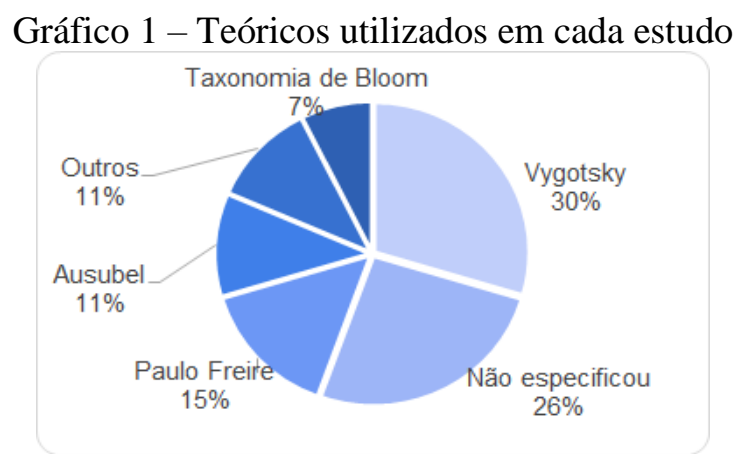

Fonte: Elaborado pelos autores, 2021.

Com relação à questão de pesquisa "Quais tecnologias digitais foram usadas?", alguns achados foram bastante relevantes (Gráfico 2). Do total de estudos selecionados, quinze são digitais e oito usam o modelo híbrido (combinação do modelo digital com não digital). Considerando os trabalhos digitais (E1 a E15) e híbridos (E16 a E23), das tecnologias utilizadas, o RPG Maker foi a ferramenta mais adotada na construção de jogos de RPG.

A comunicação digital entre os estudantes e professores durante os experimentos ocorreram também através de redes sociais (Skype, Facebook e Youtube). O WhatsApp foi a rede social mais empregada. O compartilhamento de informações ocorreu através de Ambiente Virtual de Aprendizagem (AVA), plataforma Roll20, Wordpress e com a ferramenta Google Docs. As rolagens dos dados digitais ocorreram através do rolador de dados do Roll20 apenas no estudo E21.

A interação do jogador com os demais recursos virtuais do jogo de RPG como tabuleiro, mapa, fichas de personagens e cenários ocorreu através da plataforma Roll20 no E21. Acredita-se que isso deve-se ao fato de que ela pode ser utilizada em computadores, não sendo muito usado em dispositivos móveis. Esse pode ser um dos motivos por que a maioria dos estudos utilizaram diversas tecnologias diferentes desta como: RPG Maker, AVA, celular com arquivo APK (Android Application Pack), realidade aumentada, Scratch, Wordpress e MV_Online5. Ainda sobre a interação dos jogadores com o RPG, destaca-se o estudo E4 que utilizou, com estudantes cegos, os softwares Dosvox e Jogavox no processo de aprendizagem.

Considerando a QP5 (Figura 1), percebe-se que, nos trabalhos que usaram o modelo híbrido, as tecnologias adotadas eram facilmente acessadas a partir de telefones celulares; o que apresenta este dispositivo como um recurso tecnológico alternativo para o uso de RPG em sala de aula. Identifica-se que, nesse modelo, redes sociais foram usadas em partes do jogo. Acredita-se que isso é devido ao acesso facilitado que os estudantes possuem a este tipo de ferramenta e à liberação de dados para redes sociais em alguns planos de operadoras de telefonia. A ferramenta de criação de jogos RPG mais utilizada foi o RPG Maker ${ }^{2}$, o qual contém diversos tipos de personagens que podem ou não ser controlados pelo jogador, itens, cenários e mapas. Outra ferramenta empregada na confecção de mapas de RPG foi o Inkarnate ${ }^{2}$ e, para o desenvolvimento de tabuleiro digital, foi utilizada a plataforma Roll20².

\footnotetext{
${ }^{2}$ Disponível, respectivamente, em: https://rpgmakerweb.com/, https://inkarnate.com/ e https://app.roll20.net/sessions/new.
}

V. $19 \mathrm{~N}^{\mathrm{o}} 2$, Dezembro, 2021 RENOTE 


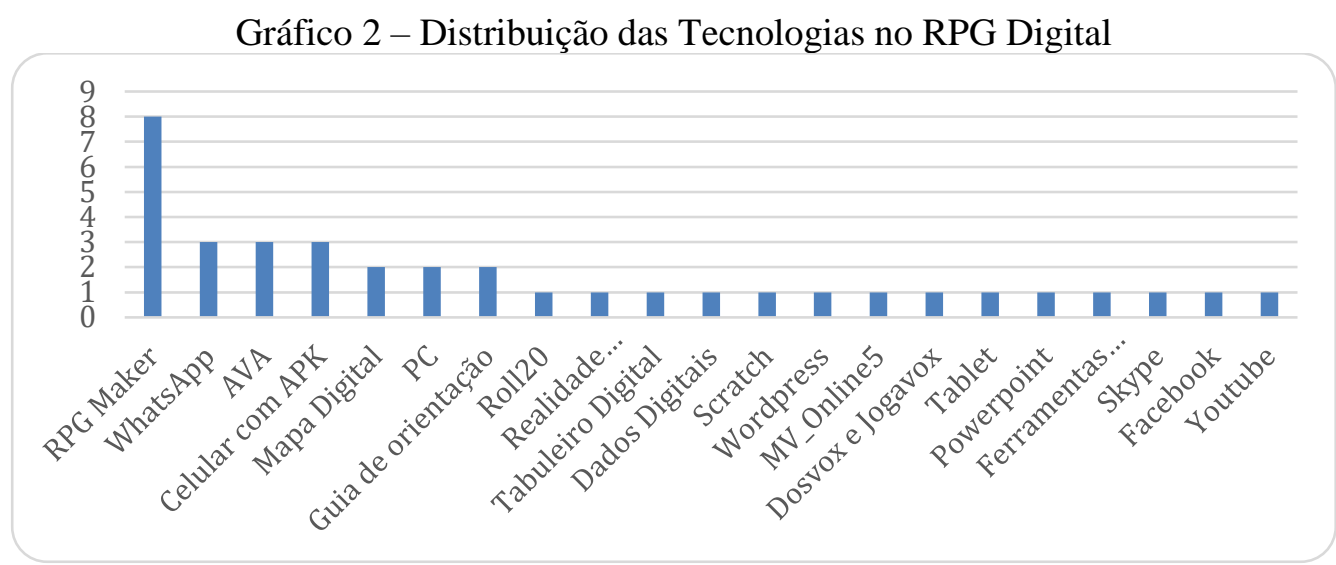

Fonte: Elaborado pelos autores, 2021.

Outras ferramentas digitais foram utilizadas. Um exemplo é o aplicativo Google Maps para pesquisa, visualização de mapas e imagens de satélite. O estudo E19 usou a linguagem de programação $\mathrm{Scratch}^{3}$ para produzir um jogo que auxilia na aprendizagem de física que pode ser jogado no computador. O Scratch foi utilizado por permitir o desenvolvimento de animações e jogos que mesclam imagem, texto e som, além de possuir uma comunidade ativa que compartilha projetos e dicas sobre o desenvolvimento de jogos.

Com a investigação realizada neste trabalho, pode-se afirmar que a maioria dos estudos selecionados apresentou o RPG no formato de jogo digital, facilitando assim, a sua aplicação por parte do docente. Esses jogos são encontrados em sites/repositórios e apresentam como grande vantagem a possibilidade do uso sem a necessidade de conhecimento específico da linguagem de programação utilizada. Por outro lado, a desvantagem encontrada ao utilizar jogos que estão disponíveis em sites ou repositórios é a sua customização ou adaptação, porque dependendo da intencionalidade do professor pode ser necessário modificar alguma funcionalidade do jogo.

Destaca-se que grande parte das tecnologias aplicadas nos estudos selecionados são gratuitas ou possuem uma versão limitada sem custos para o seu uso, o que é ideal, considerando a realidade financeira de muitas escolas públicas do Brasil. A maioria delas possui versão em português, esse é outro fator que pode auxiliar na sua utilização no contexto da sala de aula.

Considerando a questão de pesquisa " $O$ estudo utilizou narrativas específicas e interpretação de papéis?" pode-se afirmar que todos os estudos utilizaram alguma narrativa vinculada ao conteúdo em que a pesquisa foi conduzida, o que caracteriza um jogo de RPG. Os estudos E3, E8, E11, E15, E16, E18-E20 abordaram conteúdos vinculados às áreas de ciências exatas, e destacam que o fator representação de papéis contido no RPG pode auxiliar na aprendizagem de disciplinas que abordam operações matemáticas, raciocínio lógico, reações químicas, leis da física e outros conhecimentos. Além do elemento lúdico presente nas narrativas ter potencial para ser utilizado de maneira interdisciplinar, integrando conteúdos de disciplinas.

Por fim, destaca-se que no E10 fica evidenciado que o papel do professor é essencial para que a aprendizagem seja efetiva e que, para usar RPG, é importante considerar os conhecimentos prévios dos estudantes. Esse estudo, em relação aos demais selecionados, é o único que além de aplicar o jogo RPG com estudantes também desenvolveu um modelo conceitual de análise socioconstrutivista com objetivo de

\footnotetext{
${ }^{3}$ Disponível em: https://scratch.mit.edu.
} 
compreender como o RPG realiza a mediação da aprendizagem e construção cognitiva dos estudantes envolvidos no processo de aprendizagem.

$\mathrm{Na}$ maioria dos estudos analisados, as estratégias de avaliação dos conteúdos utilizaram-se de observação dos comportamentos dos participantes durante a partida. Em alguns casos, ocorreu a aplicação de questionários ou listas de exercícios antes e após a prática e apresentações em grupos (conhecimentos pré-jogo e pós-jogo). Observa-se ainda que, a maioria das avaliações considerou tanto o desempenho individual quanto o em grupo.

\section{Conclusões}

Todos os estudos analisados previamente abordam o jogo de RPG dentro do contexto educacional, detalhando os potenciais desse gênero de jogo como uma estratégia para engajar os estudantes e mobilizá-los com vistas ao processo de aprendizagem. Grando e Tarouco (2008) destacam a importância das características do RPG: "As características principais que auxiliam o jogo de RPG a se tornar uma excelente ferramenta educacional, são: socialização, cooperação, criatividade, interatividade e interdisciplinaridade" (2008, p. 8). Nos estudos aqui apontados, nota-se que o RPG pode ser aplicado em diversas áreas do conhecimento e, além de incentivar o engajamento, esse tipo de jogo potencializa as habilidades e competências apontadas na Base Nacional Comum Curricular (BNCC) como essenciais para os estudantes da educação básica.

Ao realizar o presente mapeamento, pode-se afirmar a viabilidade do professor criar ou utilizar jogos do gênero de RPG dentro do seu planejamento de aula para potencializar a chance de engajamento dos alunos. Nos estudos citados sobre a aplicação do RPG com a aprendizagem de estudantes da educação básica, fica evidente que o fator do elemento lúdico combinado com as características dos jogos RPG pode ser incluído no contexto escolar brasileiro para potencializar as chances de engajar o aluno e combater a falta de interesse e desenvolver habilidades e competências.

Em relação aos elementos clássicos utilizados em RPGs, observou-se que as versões digitais fornecem equivalência com os recursos usados em jogos não digitais, tais como: ficha de personagem ou grupo, manual de regras, guia de aplicação, mapa, bloco de anotações e demais informações que podem ser compartilhados através do Google (Google Docs). Já a rolagem de dados virtuais pode ocorrer através de aplicativos, mas pode ser utilizado o rolador de dados do Google (Roll dice ${ }^{4}$ ). O tabuleiro, cartas, marcadores e miniaturas podem ser manipulados digitalmente pela plataforma Roll20 citada anteriormente. As tecnologias relacionadas podem inclusive auxiliar o docente na aplicação desse gênero de jogo no processo de aprendizagem no momento atual, em que o ensino remoto é uma opção, devido à pandemia de Covid-19.

\section{Referências}

BITTENCOURT, J. R.; GIRAFFA, L. M. Modelando Ambientes de Aprendizagem Virtuais utilizando Role-Playing Games. Anais... Simpósio Brasileiro de Informática na Educação. Rio de Janeiro: SBC, 2003.

BRASIL. Base Nacional Comum Curricular. Brasília: MEC/Secretaria de Educação Básica, 2017. Disponível em: <http://basenacionalcomum.mec.gov.br/>. Acesso em: 15 ago. 2021.

\footnotetext{
${ }^{4}$ Disponível em: https://www.google.com/search?q=dice+roller.
} 
GALLUP. Gallup student poll 2015 results. 2016. Disponível em:

<https://www.gallup.com/topic/gallup_student_poll.aspx>. Acesso em: 12 mar. 2021.

GRANDO, A.; TAROUCO, L. O uso de Jogos Educacionais do tipo RPG na educação. RENOTE, Porto Alegre, v. 6, n.2, p. 1-10, dez., 2008.

HUIZINGA, J. Homo Ludens: o jogo como elemento da cultura. São Paulo: Perspectiva, 1993.

INSPER. Políticas Públicas para Redução do Abandono e Evasão Escolar de Jovens. Galeria de Estudos e Avaliação de Iniciativas Públicas - GESTA, 2017. Disponível em: <http://gesta.org.br/tema/engajamento-escolar/\#intro>. Acesso em: 15 out. 2021.

KELLER, J. M. Motivational Design for Learning and Performance: The ARCS Model Approach. Springer, 2009.

KITCHENHAM, B.; CHARTERS, S. Guidelines for performing systematic literature reviews in software engineering. Technical Report EBSE 2007-001. Disponível em: <https://www.elsevier.com/_data/promis_misc/525444systematicreviewsguide.pdf>. Acesso em: 11 set. 2021.

KITCHENHAM, B. Procedures for performing systematic reviews. 33, p. 1-26, 2004. UK: Keele University.

MAYO, M. R. Video games: a route to large-scale stem education? Science, v. 323, n. 5910, p. 79-82, 2009.

McGONIGAL, J. Reality is Broken: why games make us better and how they can change the world. New York: Penguin Press, 2011.

MEIRA, L.; BLIKSTEIN, P. Ludicidade, jogos digitais e gamificação na aprendizagem. Porto Alegre: Penso, 2020.

PRENSKY, M. Digital Natives, Digital Immigrants Part 1. On the Horizon. v. 9, p. 16, n. 5, Out, 2001. Disponível em:

$<$ https://www.marcprensky.com/writing/Prensky\%20-

$\%$ 20Digital\%20Natives, \%20Digital\%20Immigrants\%20-\%20Part1.pdf >. Acesso em 04 out. 2021.

SANTOS, W. O.; ISOTANI, S. Desenvolvimento de Jogos Educativos? Desafios oportunidades e direcionamentos de pesquisa. RENOTE, Porto Alegre, v. 16, n. 2, p.110, dez., 2018.

SAVI, R.; ULBRICHT, V. R. Jogos Digitais Educacionais: benefícios e desafios.

RENOTE, Porto Alegre, v. 6, n. 2, p.1-10, dez., 2008.

SOBRAL, F.; UMERES, L.; SCHANOSKI, W.; BARTELMEBS, R.; ASSIS, M. 2017. A utilização de Role Playing Games Digitais como ferramenta complementar no processo de aprendizagem de crianças deficientes visuais. Anais... Simpósio Brasileiro de Informática na Educação (SBIE).

SOBREIRA, E. S. R.; VIVEIRO, A. A.; ABREU, J. V. V. Cultura Maker e Jogos Digitais. In: MEIRA, L.; BLIKSTEIN, P. (Org.) Ludicidade, Jogos Digitais e Gamificação na Aprendizagem. Porto Alegre: Penso, 2020.

SOUZA, E. S. Uso de jogos de role playing game (RPG) como uma estratégia possível de aprendizagem de conteúdos de biologia na educação de jovens e adultos.

Interdisciplinary Scientific Journal, v. 2, n. 3, p. 384-424, 2015. 